\title{
Hydrogen Measurement during Steam Oxidation Using Coupled Thermogravimetric Analysis and Quadrupole Mass Spectrometry
}

\author{
A.J. Parkison, A.T. Nelson \\ Los Alamos National Laboratory, Los Alamos, NM 87545, United States \\ Email addresses: ajparkison@lanl.gov (A.J. Parkison), atnelson@lanl.gov (A.T. Nelson)
}

\section{Abstract:}

An analytical technique is presented with the goal of measuring reaction kinetics during steam oxidation reactions for three cases in which obtaining kinetics information often requires a prohibitive amount of time and cost. The technique presented relies on coupling thermogravimetric analysis (TGA) with a quantitative hydrogen measurement technique using quadrupole mass spectrometry (QMS). The first case considered is in differentiating between the kinetics of steam oxidation reactions and those for simultaneously reacting gaseous impurities such as nitrogen or oxygen. The second case allows one to independently measure the kinetics of oxide and hydride formation for systems in which both of these reactions are known to take place during steam oxidation. The third case deals with measuring the kinetics of formation for competing volatile and non-volatile oxides during certain steam oxidation reactions. In order to meet the requirements of the coupled technique, a methodology is presented which attempts to provide quantitative measurement of hydrogen generation using QMS in the presence of an interfering fragmentation species, namely water vapor. This is achieved such that all calibrations and corrections are performed during the TGA baseline and steam oxidation programs, making system operation virtually identical to standard TGA. Benchmarking results showed a relative error in hydrogen measurement of 5.7-8.4\% following the application of a correction factor. Finally, suggestions are made for possible improvements to the presented technique so that it may be better applied to the three cases presented.

\section{Introduction:}

The steam oxidation behavior of any cladding material to be used in nuclear reactor systems must be understood so that informed decisions may be made in materials selection and engineering controls. The ultimate goal of material selection in designing nuclear reactor systems is to prevent the release of radioactive fission products to the environment during normal conditions as well during severe accidents. Because failure of the fuel cladding is generally accepted to result in the release of much of the gaseous and volatile fission product inventory to the reactor coolant, this condition must be avoided [1-6]. Further, this has the potential to release material to the reactor containment vessel, and ultimately, to the environment.

The first line of defense in minimizing the possibility of fission product release is in ensuring the integrity of the fuel cladding is maintained. Exposure of these cladding materials to high temperatures in the presence of steam commonly results in steam oxidation reactions taking place [3-12]. The effects of these steam oxidation reactions are twofold. First, the material is oxidized, and thus, the integrity of the cladding becomes compromised due to embrittlement and loss of ductility [2-5]. It is crucial that the cladding integrity remains intact, especially during severe accidents, so understanding these degradation mechanisms is important to cladding design. The second effect is that steam oxidation reactions can produce significant volumes of potentially explosive hydrogen gas [3,4,7-11]. Perhaps nowhere was this demonstrated so dramatically as in the hydrogen explosions during the Fukushima Daiichi nuclear disaster. 
The technique presented in this paper attempts to couple mass change with hydrogen generation data so that the combined data may be used to study steam oxidation reactions. This coupling has the potential to allow one to gather continuous, real-time kinetics data on steam oxidation in the presence of gaseous impurities, steam oxidation with hydride formation, and the steam oxidation of systems with a volatile species. The method presented in this paper seeks to improve upon and complement the work performed by others to overcome many of the challenges involved in the study of steam oxidation reactions. Some of these challenges include measuring steam oxidation kinetics in the presence of gaseous impurities such as oxygen or nitrogen, measuring the steam oxidation kinetics for materials that are known to form hydrides, and determining the kinetics for systems containing a volatile oxide species.

\section{Mathematical Treatment:}

Simultaneous, real-time measurement of mass change and hydrogen generation during steam oxidation reactions has the potential to provide more information to the researcher than was previously available and in a more efficient manner. For example, it may be possible to gain access to subtle kinetics mechanism changes that would otherwise be overlooked. The technique presented in the experimental section relies on coupled Thermogravimetric Analysis (TGA) and Quadrupole Mass Spectrometry (QMS) to gather this information. Three cases are presented below which demonstrate the possible uses of such a combined technique.

\subsection{Case 1 - Competing Mass Gain Mechanisms:}

First, the situation of competing mass gain mechanisms through more than one reacting species is considered. It can be assumed that the environment seen by cladding materials during a severe accident will rarely be composed of pure steam, and that the non-steam gaseous species will affect the behavior of the material in question. Indeed, it has been found that steam oxidation of reactor materials is significantly influenced by gaseous impurities such as oxygen, nitrogen, and hydrogen [6,12-14]. Many of these studies rely on discrete data collection methods in which acquiring continuous behavior is both time consuming and the very act of observation may affect the material in unknown ways $[3,4,6-11,15,16]$. The TGA technique is attractive due to its ability to provide in situ determination of a sample's mass change during exposure to thermodynamic stimuli. Although this is far more powerful than arrested tests to examine the mass change and evolved microstructure, TGA alone does suffer from limitations.

An example is in differentiating between mass gains from oxygen through steam oxidation and mass gains from nitrogen impurities. Thermogravimetric analysis can readily discern the weight change of a sample but no detail is available regarding the cause outside of thermodynamic judgment or additional characterization. Competing mass gains may be differentiated through microscopy and spectroscopy, but such an approach inherently limits the fidelity of collected data. Further, the material must be perturbed through cooling, followed by the mounting and polishing procedure which is often required for microscopy. Barring the use of radioactive tracers, it is impossible to differentiate between mass gains from steam oxidation and mass gains from oxidation by molecular oxygen impurities. It therefore becomes difficult to determine how the steam oxidation reaction kinetics and mechanisms are changed by the addition of these species. 
In order for this coupled technique to be applicable for this case, it must be assumed that all hydrogen measured using QMS is produced through steam oxidation, and that one mole of atomic oxygen is added to the sample material for every mole of molecular hydrogen measured. This assumption is reasonable for testing conditions which are relatively pure, and for which hydride formation contributes little to the sample mass gain.

The mass gain caused by steam oxidation and impurities may be individually calculated by starting with the following equation;

$$
\text { Equation 1: } \quad \Delta m(T G A)=\Delta m(X)+\Delta m(Q M S)
$$

Where $\Delta \mathrm{m}(\mathrm{TGA})$ is the mass gain as measured by the TGA instrument, in this case $\Delta \mathrm{m}(\mathrm{X})$ is the mass gain from impurities such as oxygen and nitrogen, and $\Delta \mathrm{m}(\mathrm{QMS})$ is the mass gain from the steam oxidation reaction as calculated by the QMS hydrogen measurement using the assumptions mentioned above. The $\Delta \mathrm{m}(\mathrm{QMS})$ term is determined by measuring the molar flow rate using QMS. The full procedure is presented in a later section, but the basic equation is shown as Equation 2, where $\mathrm{M}_{0}$ is the molar mass of atomic oxygen. This equation may also be interpreted as being the mass gain due to steam oxidation for this first case.

Equation 2: $\quad \Delta m(Q M S)=\operatorname{mol}\left(H_{2}\right) \times M_{O}$

Combining Equation 1 and Equation 2 results in an equation corresponding to the mass gain caused by impurities.

$$
\text { Equation 3: } \quad \Delta m(X)=\Delta m(T G A)-\operatorname{mol}\left(H_{2}\right) \times M_{O}
$$

\subsection{Case 2 - Oxide and Hydride Formation:}

The next situation to be considered is that in which there are competing oxide and hydride mass gain mechanisms. Study of steam oxidation behavior in zirconium alloys is complicated due to zirconium's affinity for hydrogen [2,8-10,17]. The formation of hydrides in these materials is important in understanding and ensuring the mechanical integrity of the material during a severe nuclear accident. It is widely accepted that formation of hydride platelets is a major contributor to the degradation of the cladding's mechanical properties, and thus, its ability to prevent release of radioactive fission products $[8,17]$. While it is indeed possible to determine hydride formation rates during steam oxidation through microscopy or spectroscopic techniques, these inherently produce discrete data sets. The technique presented here attempts to allow one to independently study both the oxide and hydride formation in these alloys during steam oxidation reactions. For this case, the $\Delta \mathrm{m}(\mathrm{X})$ term in Equation 1 is a sum of mass gains from both oxide and hydride formation in the material, as can be seen in Equation 4. This treatment is necessary because hydride formation will clearly reduce the amount of hydrogen measured using QMS, and thus reduce the calculated oxide growth during steam oxidation.

Equation 4: $\quad \Delta m(X)=\Delta m($ hydride $)+\Delta m($ oxide $)$

The hydride and oxide terms in Equation 4 must be present in ratios equal to the molar ratios of hydrogen and oxygen in water. Equation 4 may then be expressed as, 
Equation 5: $\quad \Delta m(X)=\Delta m($ hydride $) \times\left[1+\frac{M_{O}}{M_{H_{2}}}\right]$

Combining the equations above results in an expression for hydride formation using known or directly measureable quantities.

Equation 6: $\quad \Delta m($ total hydride $)=[\Delta m(T G A)-\Delta m(Q M S)] \times\left[\frac{1}{1+\frac{M_{O}}{M_{H_{2}}}}\right]$

Further, an expression for total oxide formation may be derived.

Equation 7: $\quad \Delta m($ total oxide $)=\Delta m(T G A) \times\left[1-\frac{1}{1+\frac{M_{O}}{M_{H_{2}}}}\right]+\Delta m(Q M S) \times\left[\frac{1}{1+\frac{M_{O}}{M_{H_{2}}}}\right]$

\subsection{Case 3 - Volatile Oxide:}

The last and most complicated situation considered here is steam oxidation of materials that yield a volatile oxide. There are some materials of interest whose steam oxidation kinetics have been elusive due to formation of volatile oxides. An example of such materials are tungsten, molybdenum, and their alloys $[13,18]$. For the sake of illustration, the steam oxidation of molybdenum will be discussed here. Considering steam oxidation of these materials have both mass loss and mass gain mechanisms, care must be taken to ensure that the TGA data is interpreted correctly $[13,18,19]$. Oxidation of molybdenum results in both a mass gain and a mass loss term above $600{ }^{\circ} \mathrm{C}$ due to formation of $\mathrm{MoO}_{2}$ from the metal as well as formation of $\mathrm{MoO}_{3}$ from $\mathrm{MoO}_{2}$, respectively. Use of TGA alone to characterize this oxidation reaction will produce a single mass gain or mass loss term. Detailed analysis of the oxidized sample is necessary to differentiate between the oxidation behaviors for the two oxides. Use of the coupled TGA-QMS approach facilitates measurement of the decoupled kinetics of these mechanisms.

This third case assumes that steam oxidation is the only reaction taking place, with no competition from oxygen and nitrogen impurities or hydride formation. It also assumes that the volatilization rate of $\mathrm{MoO}_{3}$ is high enough such that a negligible amount remains in the material. Care must be taken to ensure that this final assumption is true by considering both the temperature and flow rate of the system.

For this third case, the terms in Equation 1 assume different definitions dependent on the stoichiometry of the Mo-O system. The basic reactions taking place during the steam oxidation of Mo are shown below.

Equation 8: $\quad \mathrm{Mo}+2 \mathrm{H}_{2} \mathrm{O}=\mathrm{MoO}_{2}+2 \mathrm{H}_{2}$

Equation 9: $\quad \mathrm{MoO}_{2}+\mathrm{H}_{2} \mathrm{O}=\mathrm{MoO}_{3}+\mathrm{H}_{2}$

(Note: The volatile species of greatest concern for Mo steam oxidation is molybdic acid $\mathrm{MoO}_{3} \mathrm{H}_{2} \mathrm{O}$, or some variation thereof $[18,20]$. For the sake of simplicity, and because the stoichiometry is identical for our purposes, the trioxide will be used here.) 
The first term of Equation 1, $\Delta \mathrm{m}(\mathrm{TGA})$, is the difference between the mass gain due to oxidation of $\mathrm{Mo}$ and the mass loss through volatilization of $\mathrm{MoO}_{3}$,

Equation 10: $\Delta m(T G A)=\Delta m\left(O_{I V}\right)-\Delta m\left(M o_{V I}\right)$

Where $\Delta \mathrm{m}\left(\mathrm{O}_{\mathrm{IV}}\right)$ is the mass gain from oxygen through formation of the non-volatile $\mathrm{MoO}_{2}$, and $\Delta \mathrm{m}\left(\mathrm{MovI}_{\mathrm{vI}}\right)$ is the mass loss of Mo through volatilization of $\mathrm{MoO}_{3}$. Even though TGA alone is unable to differentiate between these mass gain/loss rates, simultaneous measurement of hydrogen allows access to these rates.

The third term of Equation 1, $\Delta \mathrm{m}(\mathrm{QMS})$, is a measure of the total oxygen which has been added to the Mo system by steam oxidation through formation of both the dioxide (IV) and trioxide (VI).

Equation 11: $\quad \Delta m(Q M S)=\Delta m\left(O_{I V}\right)+\Delta m\left(O_{V I}\right)$

where $\Delta \mathrm{m}\left(\mathrm{O}_{\mathrm{VI}}\right)$ is the amount of oxygen in the volatile $\mathrm{MoO}_{3}$ species and $\Delta \mathrm{m}\left(\mathrm{O}_{\mathrm{IV}}\right)$ is defined above. Equation 10 and Equation 11 may be solved through knowledge of the stoichiometry of the system, in this case, the stoichiometry of $\mathrm{MoO}_{3}$.

Equation 12: $\quad \frac{3 M_{O}}{M_{M o}}=\frac{\Delta m\left(O_{V I}\right)}{\Delta m\left(M o_{V I}\right)}$

Combining the above equations, the amount of Mo that has been volatilized from the material may be expressed as,

Equation 13: $\quad \Delta m\left(M o_{V I}\right)=\frac{\Delta m(Q M S)-\Delta m(T G A)}{1+\frac{3 M_{0}}{M_{M o}}}$

the amount of oxygen remaining in the sample as $\mathrm{MoO}_{2}$ may be expressed as,

Equation 14: $\Delta m\left(O_{I V}\right)=\frac{\Delta m(Q M S)+\Delta m(T G A) \times \frac{3 M_{O}}{M_{M o}}}{1+\frac{3 M_{O}}{M_{M o}}}$

and for the sake of completeness, the amount of oxygen in the volatilized $\mathrm{MoO}_{3}$ is

Equation 15: $\Delta m\left(O_{V I}\right)=\frac{[\Delta m(Q M S)-\Delta m(T G A)] \times \frac{3 M_{O}}{M_{M o}}}{1+\frac{3 M_{O}}{M_{M o}}}$

Practical applications would often be concerned with the total material consumption rate. This consumption may be expressed as the sum of $\mathrm{MoO}_{2}$ and $\mathrm{MoO}_{3}$ formation. The mass of material compromised through $\mathrm{MoO}_{3}$ formation is simply given by Equation 13, while the mass of material compromised through $\mathrm{MoO}_{2}$ formation may be derived using Equation 14 and by knowing the stoichiometry of the system. 
Equation 16: $\Delta m\left(M o_{I V}\right)=\Delta m\left(O_{I V}\right) \times \frac{M_{M o}}{2 M_{O}}$

Combining Equation 13, Equation 14, Equation 15and Equation 16 gives an expression for the consumption of Mo in units of mass.

Equation 17: Mo consumption $=\frac{\Delta m(Q M S) \times\left[1+\frac{3}{2} \times \frac{M_{M o}}{3 M_{O}}\right]+\Delta m(T G A) \times \frac{1}{2}}{1+\frac{3 M_{O}}{M_{M o}}}$

Before any of the above cases may be considered, a method of quantitative measurement of hydrogen generation must be developed so that the time-dependent $\operatorname{mol}\left(\mathrm{H}_{2}\right)$ term from Equation 2 may be measured. Measurement of this term was attempted with a commercial QMS system, and is presented below.

\section{Experimental Approach:}

The first difficulty one encounters when attempting to measure hydrogen in the presence of water vapor using QMS is that water vapor gives a mass to charge $(\mathrm{m} / \mathrm{z})$ fragmentation pattern of 18,17 , 16 , and 2 . This becomes problematic because hydrogen also produces an $\mathrm{m} / \mathrm{z}$ signal of 2 . It therefore becomes necessary to account for this background 2 signal produced by the water vapor so that one may measure hydrogen generation. Typically, whenever interference due to fragmentation is a concern it is accounted for by measuring calibration gasses and then setting up and solving the resulting matrix. However, it is often desirable to introduce steam while the material of interest is already at temperature. This rapid change in atmosphere renders any steady-state measurement of calibration gasses inaccurate. A technique will be presented here that attempts to allow one to correct for the 2 fragmentation caused by water through measurement of the time-dependent $18 / 2 \mathrm{~m} / \mathrm{z}$ ratio using QMS during the TGA baseline program, and then applying this ratio to the steam oxidation program. The result is a corrected signal attributable only to molecular hydrogen.

\subsection{Basic Theory:}

The $2 \mathrm{~m} / \mathrm{z}$ signal caused by water must be accounted for if one desires to quantitatively measure hydrogen in the presence of water vapor. It was noticed during testing that the 18 and 2 signals would drift from run to run, likely through SEM drift. In other systems, this drift would be corrected through measurement of a zero gas and known calibration gasses. Software is often used to set up and solve a matrix, thus correcting for any interference between species. This standard method relies on the ion current ratios between species being constant during the period of interest. However, it was found that the 18/2 ratio was not constant even during baseline runs, as seen in Figure 1. This is believed to be due to differing turbo pump efficiencies when removing hydrogen compared to other species. The hydrogen is hypothesized to accumulate within the mass spectrometer high-vacuum system until it reaches a steady state condition. This limits the usefulness of standard calibrations which rely on near steady state operation. The research presented here assumed a demand for an instantaneous insertion of steam while the sample was already at temperature. Therefore, an alternative correction technique was developed. 
While the $18 / 2$ ratio was not constant during runs, this time-dependent ratio was seen to vary little between runs. This suggests that an 18/2 ratio measured during a TGA baseline run, for example, could be applied toward the QMS signal measured during a TGA steam oxidation run. Essentially, the 18/2 ratio measured during the TGA baseline is a measure of the background hydrogen signal provided by water as a function of time. The corrected 2 signal attributable to hydrogen produced during steam oxidation can thus be calculated through the following equation,

Equation 18: $\quad 2_{C o r}(t)=2_{S O}(t)-\frac{18_{S O}(t)}{18 / 2_{B L}(t)}$

Where $18_{\mathrm{so}}(\mathrm{t})$ and $2_{\mathrm{so}}(\mathrm{t})$ are the 18 and 2 ion currents as measured during the TGA steam oxidation program, and the $18 / 2_{\mathrm{BL}}(\mathrm{t})$ term is the time-dependent ratio of the 18 and 2 ion currents as measured during the TGA baseline program. Equation 18 results in an ion current that corresponds only to hydrogen formed through steam oxidation. All ion currents must first be corrected for background as will be described in the next section.

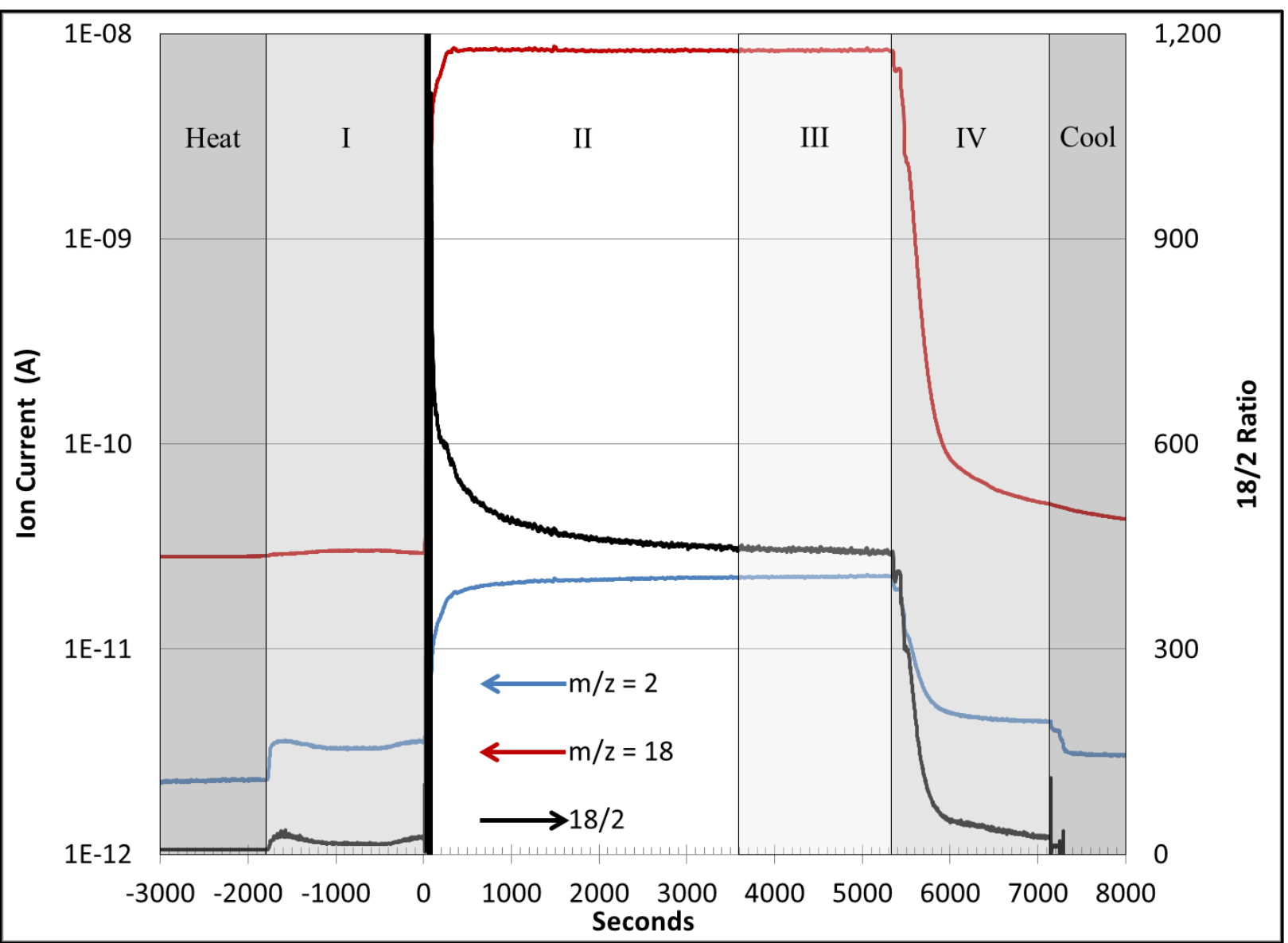

Figure 1: Ion currents for $\mathrm{m} / \mathrm{z}$ of 2 and 18 seen alongside the corrected 18/2 ratio. Instantaneous insertion of steam results in an $18 / 2 \mathrm{~m} / \mathrm{z}$ ratio that is not constant, thus requiring alternate means of correcting for the background 2 signal caused by the presence of water vapor. 


\subsection{Zero Gas Measurement:}

In order for the theory presented above to be valid, it is necessary to measure and correct for background ion current, or zero-gas. The zero-gas measurement must be measured both before and after the steam oxidation reaction due to SEM drift. This is especially true when measuring trace amounts of hydrogen, as is often the case with steam oxidation reactions. The result of this before and after zero-gas measurement is a zero-gas measurement in the form of a linear function, seen in Equation 19, to be subtracted from the measured time-dependent 18 and 2 ion currents during the baseline and steam oxidation TGA programs. The zero-gas measurements take place during isothermal holds located before and after steam is introduced into the system for both the baseline and steam oxidation programs. This can be seen in Figure 3.

Equation 19: $\quad Z G(t)=Z G_{i}+t \times \frac{Z G_{f}-Z G_{i}}{\Delta t}$

The $\mathrm{ZG}(\mathrm{t})$ term is the zero-gas value to be subtracted from the measured ion currents as a function of time, $\mathrm{ZG}_{\mathrm{i}}$ and $\mathrm{ZG}_{\mathrm{f}}$ are the zero-gas measurements during the TGA baseline and steam oxidation runs prior to and after the introduction of steam, and $\Delta \mathrm{t}$ is the time between zero-gas measurements.

\subsection{Span Gas Measurement:}

After subtracting the background ion current and then applying Equation 17, the now corrected ion current can be correlated to known concentrations of hydrogen. This span-gas measurement is performed using a known $\mathrm{Ar}-\mathrm{H}_{2}$ calibration gas immediately following the steam oxidation reaction so as not to perturb the material prior to steam oxidation, but before the final zero-gas measurement. The ion current measured during the span-gas measurement must also be corrected for background using the zero-gas function and the interference due to fragmentation of steam by Equation 19 and Equation 18, respectively.

It is important that the total volumetric flow rate during the span-gas measurement is identical to the volumetric flow rate during the steam oxidation reaction. This can be accomplished by flowing an Ar carrier gas during the steam oxidation reaction, and then replacing this Ar carrier gas with an $\mathrm{Ar}-\mathrm{H}_{2}$ calibration gas during the span-gas measurement. This results in an ion current that corresponds to a hydrogen flow rate, thus eliminating the need to know the total system flow rate, vapor quality, or temperature. Instead, all that is needed is the temperature of the Mass Flow Controller (MFC) responsible for insertion of $\mathrm{Ar}$ and $\mathrm{Ar}-\mathrm{H}_{2}$, which will likely be maintained at room temperature. The volumetric flow rate, and thus, the hydrogen production at any point in time may be found using the following equation,

Equation 20: $\quad Q(t)=2_{C o r}(t) \times \frac{Q(S G)}{2_{S G}}$

where $Q(t)$ is the volumetric flow rate of hydrogen during the steam oxidation reaction, $Q(S G)$ is the volumetric flow rate of hydrogen during the span-gas measurement, and $2_{\mathrm{SG}}$ is the ion current during the span-gas measurement after being corrected using Equation 19. The molar production rate, $\operatorname{mol}\left(\mathrm{H}_{2}\right)$, can then easily be calculated. 
Equation 21: $\operatorname{mol}\left(H_{2}\right)=Q(t) \times \frac{P}{T \times R}$

Where $\mathrm{R}$ is the gas constant, $\mathrm{P}$ is atmospheric pressure, and $\mathrm{T}$ is room temperature, assuming that the MFC is maintained at atmosphere and pressure. The local atmospheric pressure and temperature were assumed to be 0.7687 atm and $77^{\circ} \mathrm{C}$, respectively.

The $\Delta \mathrm{m}(\mathrm{QMS})$ term then becomes,

Equation 22: $\Delta m(Q M S)=2_{C o r}(t) \times \frac{Q(S G)}{2_{S G}} \times \frac{P}{T \times R} \times M_{O}$

\section{Experimental Procedure:}

The sample mass change and hydrogen generation rates during steam oxidation reactions were measured using a simultaneous thermal analyzer (STA 449 F3, Netzsch Instruments, Selb, Germany) equipped with a water vapor furnace whose exhaust was passed by a quadrupole mass spectrometer (QMS 403 D Aeolos, Netzsch Instruments, Selb, Germany). Steam was provided using a high precision vapor generator (ASTEAM DV2MK, Bronkhorst High Teck Instruments, Ruurio, The Netherlands), and argon carrier gas was supplied after being passed through a copper oxygen getter (2A, Centorr Vacuum Industries, Nashua, New Hampshire). The partial pressure of oxygen in the argon carrier gas was maintained below $10^{-12} \mathrm{ppm}$, and was monitored at both the inlet and outlet of the water vapor furnace using two zirconia oxygen sensors (RapidOx OEM447, Cambridge Sensotec, St. Ives, United Kingdom). The samples were located on an alumina platform which sat atop a Type-S thermocouple.

Two sample compositions were studied: zirconium foil and T91 steel plates. The zirconium foils had a nominal length, width, and thickness of $25.4 \mathrm{~mm}, 6.35 \mathrm{~mm}$, and $0.127 \mathrm{~mm}$, respectively. These foils were bent in the middle so that they could be stood on end, thus exposing both surfaces to the steam atmosphere. The T91 Steel plates had nominal dimensions of $9.54 \mathrm{~mm}, 9.54 \mathrm{~mm}$, and $1.55 \mathrm{~mm}$. A platinum wire was used to stand the plates upright during the steam oxidation reaction to ensure exposure of both sides to the steam environment. The sample configurations can be seen in Figure 2. 


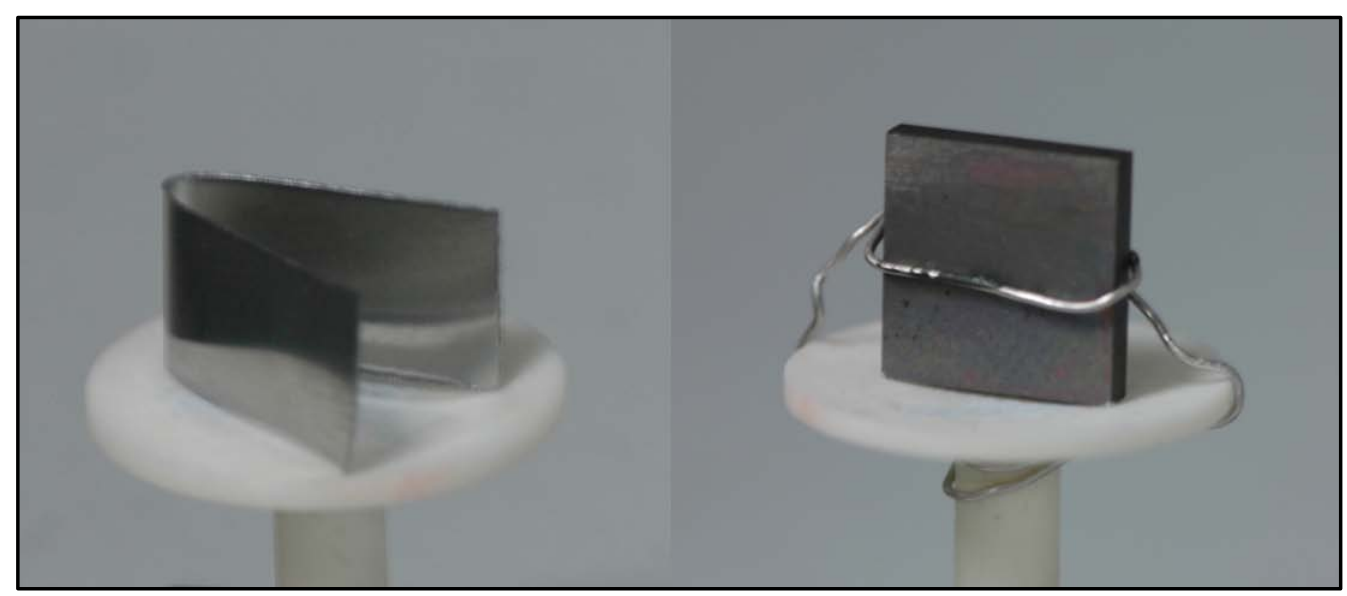

Figure 2: Geometry of the foil (left) and plate (right) samples. These orientations were used to ensure a consistent surface area is exposed to steam. The plate samples are held in place by a platinum wire.

\subsection{TGA Programs:}

Accurate measurement of hydrogen in the presence of steam requires a multistage TGA program in order to acquire the data needed to satisfy the method presented above. During each of these baseline and steam oxidation programs, several important pieces of information are measured in a single pass. This is to reduce the effect of SEM drift between sample runs, so that reliable hydrogen measurements may be obtained. The TGA program can be seen in Figure 3 for both the baseline and steam oxidation programs.

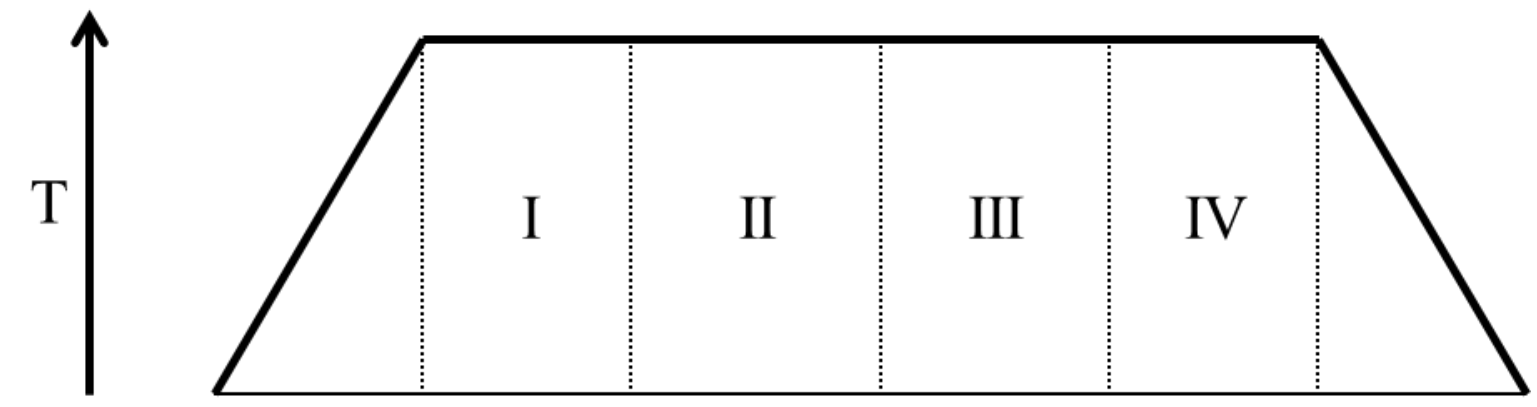

\begin{tabular}{|c|c|c|c|c|c|c|}
\hline SO & Heat Up & $\begin{array}{c}\text { Initial Zero } \\
\text { Gas }\left(\mathrm{ZG}_{\mathrm{i}}\right)\end{array}$ & Steam Oxidation & Span Gas (SG) & $\begin{array}{c}\text { Final Zero } \\
\left.\text { Gas }_{(\mathrm{ZG}}\right)\end{array}$ & Cool Down \\
\hline $\begin{array}{c}\text { Steam } \\
(\mathrm{g} / \mathrm{h})\end{array}$ & 0 & 0 & 5 & 5 & 0 & 0 \\
\hline $\begin{array}{c}\text { Carrier Gas } \\
(\mathrm{ml} / \mathrm{min})\end{array}$ & 0 & 0 & 50 & 50 & 0 & 0 \\
\hline $\begin{array}{c}\text { Protective Gas } \\
(\mathrm{ml} / \mathrm{min})\end{array}$ & 220 & 220 & 20 & 20 & 220 & 220 \\
\hline $\begin{array}{c}\text { Purge Gas } \\
(\mathrm{ml} / \mathrm{min})\end{array}$ & 135 & 135 & 100 & 100 & 135 & 135 \\
\hline $\begin{array}{c}\text { Ar-6\% } \mathrm{H}_{2} \\
(\mathrm{ml} / \mathrm{min})\end{array}$ & 0 & 2 & 2 & 2 & 0 & 2 \\
\hline
\end{tabular}


Figure 3: Schematic of the TGA baseline program showing the order and flow conditions of the various steps used during these experiments for hydrogen measurement in steam. Split boxes indicate differences between the baseline (BL) and steam oxidation (SO) programs.

\subsection{Baseline:}

In addition to buoyancy correction, the TGA baseline program is used to measure the timedependent $18 / 2$ ratio. All ion currents collected during the steam oxidation program are corrected according to Equation 18 using the 18/2 ratio measured during the baseline program. The four regions seen in Figure 3 for the baseline program are described below.

Step I: This first step depicted in Figure 3 corresponds to a 30 minute hold at temperature prior to the introduction of steam. The initial zero-gas $\left(\mathrm{ZG}_{\mathrm{i}}\right)$ used in Equation 19 for correcting the 18/2 ratio is collected at this time for the baseline run. The $2 \mathrm{ml} / \mathrm{min}$ hydrogen flow during this step exists because traces of a reducible oxide, namely $\mathrm{MoO}_{3}$, existed in the system used for these tests. This may not be necessary for clean systems which do not contain reducible oxides. Regardless, a low hydrogen background will not affect the measured hydrogen for Cases 1 and 3, with the exception of Case 1 with trace oxygen. A pure system without a low hydrogen background will be needed for Case 2 studies.

Step II: Steam is introduced and the measured 18 and 2 ion currents produce the $18 / 2$ ratio used in Equation 18 after correcting for the zero-gas measurements. The volumetric flow rate due to insertion of steam and its carrier gas is accounted for by a decrease in flow rate of the Ar purge and protective gasses. This decrease is set to be approximately equal to the sum of the steam and its carrier gas so that the QMS is sampling a near-constant volumetric flow rate throughout the run. Matching the exact steam flow rate is not crucial here; an approximation is likely sufficient. This is because the most important quantity, the span gas, is measured under the same flow conditions that are seen during the steam oxidation reaction.

Step III: The 18/2 ratio continues to be measured. This $18 / 2$ ratio is later applied to Step III of the steam oxidation run using Equation 18 to correct the span-gas $\left(2_{\mathrm{SG}}\right)$ measurement to be used in Equation 20. Again, the 18 and 2 ion currents are first corrected based on the zero-gas measurements gathered during Steps I and IV prior to producing the 18/2 ratio.

Step IV: The final zero-gas $\left(\mathrm{ZG}_{\mathrm{f}}\right)$ for use in correcting the 18 and 2 ion currents according to Equation 19 for use in the $18 / 2$ ratio is measured here. The purge and protective gasses are increased back to their original values to account for the end of steam flow.

\subsection{Steam Oxidation:}

The steam oxidation program is used to measure the 2 and 18 ion currents. Along with the 18/2 ratio determined during the baseline program, the 2 and 18 ion currents are used to calculate the corrected $(2$ cor $)$ hydrogen ion current according to Equation 18. The initial and final zero-gasses for the steam oxidation run are measured, along with the span-gas.

Step I: Again, this is a 30 minute hold at temperature, and is used to measure the initial zero-gas $\left(\mathrm{ZG}_{\mathrm{i}}\right)$ value for use in Equation 19. This is then used to correct the 2 and 18 ion currents measured both during the steam oxidation reaction and the span-gas measurement. 
Step II: The steam oxidation reaction takes place during this step. The 2 and 18 ion currents are collected, later to be corrected using the zero-gas measurements collected during Steps I and IV of the steam oxidation run and the 18/2 ratio collected during Step II of the baseline run. Again, the gas flow rates are altered according to the description provided for the baseline run.

Step III: The span gas is measured during this step. The Ar purge gas is replaced with an $\mathrm{Ar}-\mathrm{H}_{2}$ calibration gas at an identical volumetric flow rate. This value is corrected based on the zero-gas measurements gathered in Steps I and IV of the steam oxidation run, as well as the 18/2 ratio measured in Step III of the baseline run.

Step IV: The final zero-gas $\left(\mathrm{ZG}_{\mathrm{f}}\right)$ for use in correcting both the 2 and 18 ion currents during the steam oxidation reaction and the span-gas measurement is collected during this step

\subsection{QMS Settings:}

The QMS was operated using the electron multiplier at a bias voltage of 1050 . Even though the electron multiplier is more susceptible to signal drift, this mode was found to be superior to the built-in Faraday collector when measuring trace amounts of hydrogen.

An intelligent balance must be reached between time resolution and accuracy because QMS is a scanning technique. The sharp transients seen in these experiments drive the need for a short scan time so that an accurate 18/2 ratio may be measured. A scan time that is too long during the first few minutes, when the 18/2 ratio has the greatest rate of change, will result in a somewhat decoupled 18/2 ratio. This would, in turn, produce an inaccurate corrected hydrogen ion current once Equation 18 is applied. Conversely, a longer scan time is needed for detection of trace amounts of hydrogen near the end of the steam oxidation reaction. A scan time of 1 second per species was found to provide an acceptable balance between time resolution and accuracy during these steam oxidation reactions. The peak resolution was set to 50 out of 256 so that trace amounts of hydrogen may be measured with minimal interference from the background ion current. For this piece of equipment, a peak resolution of 50 means that the middle $\sim 20 \%$ of the $\mathrm{m} / \mathrm{z}$ span will be counted.

\section{Experimental Results:}

The goal of these experiments is to demonstrate the capabilities and limitations of the technique presented above. To this end, zirconium foils and T91 steel (Fe-9Cr-1Mo) plates were selected as benchmarking materials in an attempt to quantify and differentiate between the sources of error which one may encounter if this technique is to be applied using a similar system by future researchers. These materials were selected for two reasons. First, they are known to display very different steam oxidation performance. Zirconium is known to display parabolic kinetics, and T91 steel has been shown to display linear steam oxidation kinetics. Second, the physical geometry of the materials are very different as seen in Figure 2. If this technique were to be applied to other systems, it is important to understand the effect that these two variables might have on the quantification of hydrogen generation.

\subsection{Baseline Measurement:}

In order for the experiments presented below to be interpreted correctly, it is necessary to understand the magnitude and nature of the error acquired during the baseline program. Three 
identical baseline measurements were performed at $1000^{\circ} \mathrm{C}$ to make this determination. The 2,18 , and $18 / 2$ peaks and ratio measured for these three identical baseline measurements were then compared to one another, producing a reasonable estimate for the systematic error that one should expect for these baseline measurements.

It may be seen in Figure 4 that the systematic error is prohibitively high upon initial insertion of steam. The cause of this is likely due to the need for steam to travel from the water vapor generator, through a heated transfer line, and finally through the water vapor furnace before reaching the sample. This introduces a substantial variation in timing between the baseline program and the steam oxidation program. While it may be possible to decrease this variability through increasing the flow rate of the steam carrier gas, this would effectively dilute the hydrogen. Dilution of the hydrogen will increase the effective hydrogen detection limit as well as increase the relative error of the hydrogen measurement. Unfortunately, this initial error is present during what is perhaps the most important region for quantitative measurement of hydrogen production for many materials of interest, zirconium being one of them. Following this initial period of large systematic error, the relative error for the 2,18 , and $18 / 2$ peaks and ratio go to $2.6,4.7$, and $5.3 \%$, respectively. These are reasonable estimates for the error that one should expect when comparing the QMS signals obtained during the TGA programs to one another, including when applying the baseline runs to the steam oxidation runs as is done when applying Equation 18.

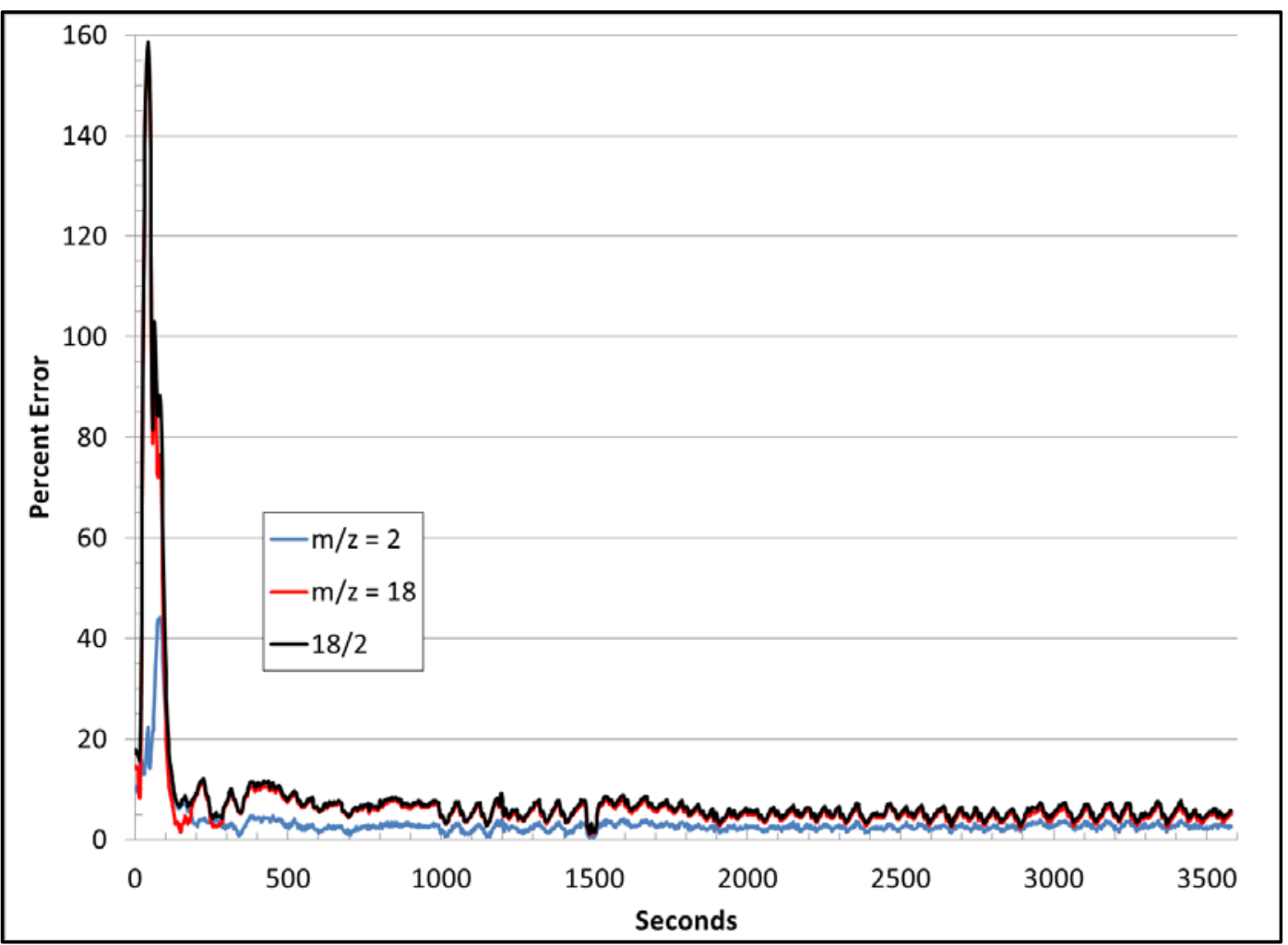

Figure 4: Systematic error for the 2,18 , and 18/2 signals. This data corresponds to Step II of the baseline program.

\subsection{Zirconium Foil Benchmarking:}


Five steam oxidation tests were performed on zirconium foil samples. All five foils were exposed to $1000^{\circ} \mathrm{C}$ steam according the program depicted in Figure 3. The measured TGA signal was compared to the mass gain as calculated through hydrogen measurement using QMS to demonstrate the repeatability of the technique. It was found that the mass gain as calculated through hydrogen measurement contained a $6.0 \%$ relative error among these five samples, while that measured using TGA showed a $2.4 \%$ relative error. The relative error of the hydrogen measurement technique is clearly the limiting factor from an error propagation perspective. Any improvements in decreasing this variability will surely improve the applicability of the coupled technique. Much of this error is incurred through the simple act of measurement using QMS. This can be seen in the previous section which presents the relative error of the baseline 18/2 ratio as only slightly less than the error obtained after following the procedure and technique outlined above. This suggests that there remains much room for optimization of the QMS parameters, as well as suggesting that the error introduced through the coupled measurement technique is minimal. The calculated hydrogen generation rate may be seen in Figure 5 alongside the raw 18 and 2 ion currents.

The mass gain calculated through hydrogen measurement was seen to differ significantly from that measured using TGA, as can be seen in Figure 6 and Table 1. A possible source of this error may be similar to the assumed cause of the time dependent $18 / 2$ ratio as described previously. During a rapid release of hydrogen, the efficiency of the turbo pump to remove this hydrogen may affect the measured results. If this hypothesis is correct, it would imply that the measured time-dependent concentration of hydrogen would be strongly dependent on the magnitude and shape of the hydrogen pulse formed through steam oxidation. This would lead one to conclude that the technique presented above is questionable for this specific equipment, but this was not proven. Improved accuracy can be attained by use of a correction factor which may be applied to the hydrogen calculation, resulting in an agreement with the TGA measurement of within $5.7 \%$. The correction factors calculated for each zirconium foil run are shown in the last column of Table 1. This seems to indicate that once the correction factor is applied, the error of the coupled technique is dominated by the error in the hydrogen measurement itself. This reiterates the point that improvements in the precision of the hydrogen measurement will benefit the coupled technique. It may be possible to improve upon this precision through systematically optimizing the gas flow rates, scan times, and the peak resolution parameter within the QMS software.

Table 1: Tabulated comparison between mass gains measured using TGA and those calculated using hydrogen generation data. These zirconium foils were reacted in $1000^{\circ} \mathrm{C}$ steam for one hour.

\begin{tabular}{ccccc}
\hline Sample & $\begin{array}{c}\text { Starting Mass } \\
(\mathrm{mg})\end{array}$ & $\begin{array}{c}\text { TGA Mass Gain } \\
(\mathrm{mg})\end{array}$ & $\begin{array}{c}\text { Hydrogen Calculation } \\
(\mathrm{mg})\end{array}$ & $\begin{array}{c}\text { Correction } \\
\text { Factor }\end{array}$ \\
\hline $\mathrm{Zr}-1$ & 133.32 & 36.36 & 27.9 & 1.30 \\
\hline $\mathrm{Zr}-2$ & 134.03 & 37.79 & 31.1 & 1.22 \\
\hline $\mathrm{Zr}-3$ & 131.34 & 36.87 & 29.8 & 1.24 \\
\hline $\mathrm{Zr}-4$ & 132.59 & 35.55 & 28.4 & 1.25 \\
\hline $\mathrm{Zr}-5$ & 132.41 & 35.86 & 32.1 & 1.11 \\
\hline Mean & & 36.49 & 29.9 & $1.22 \pm 0.07$
\end{tabular}




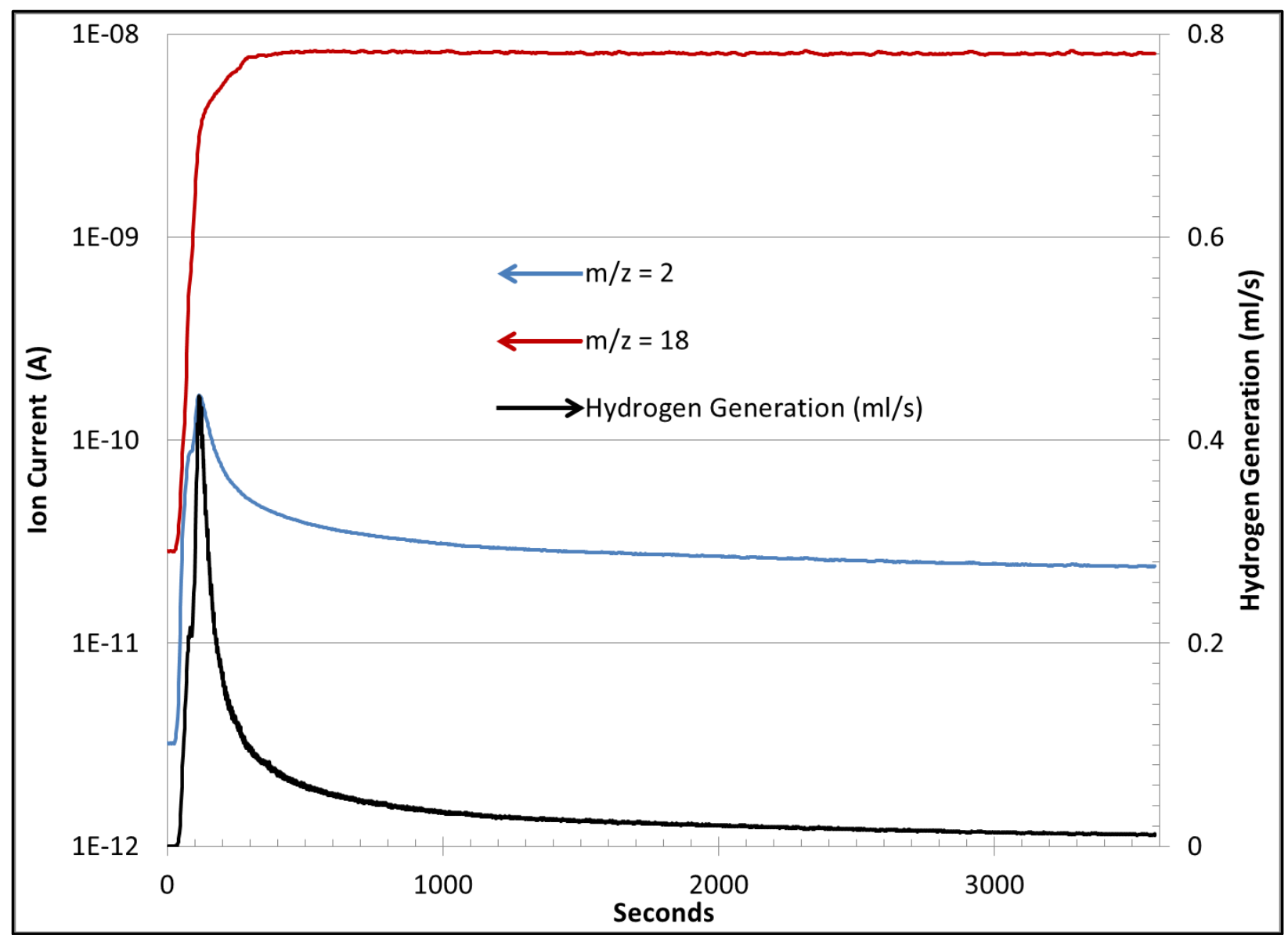

Figure 5: Ion currents for $\mathrm{m} / \mathrm{z}$ of 2 and 18 seen alongside the hydrogen generation rate as determined through QMS measurement. Displayed data is the average of the five zirconium foil samples reacted in $1000{ }^{\circ} \mathrm{C}$ steam for one hour. 


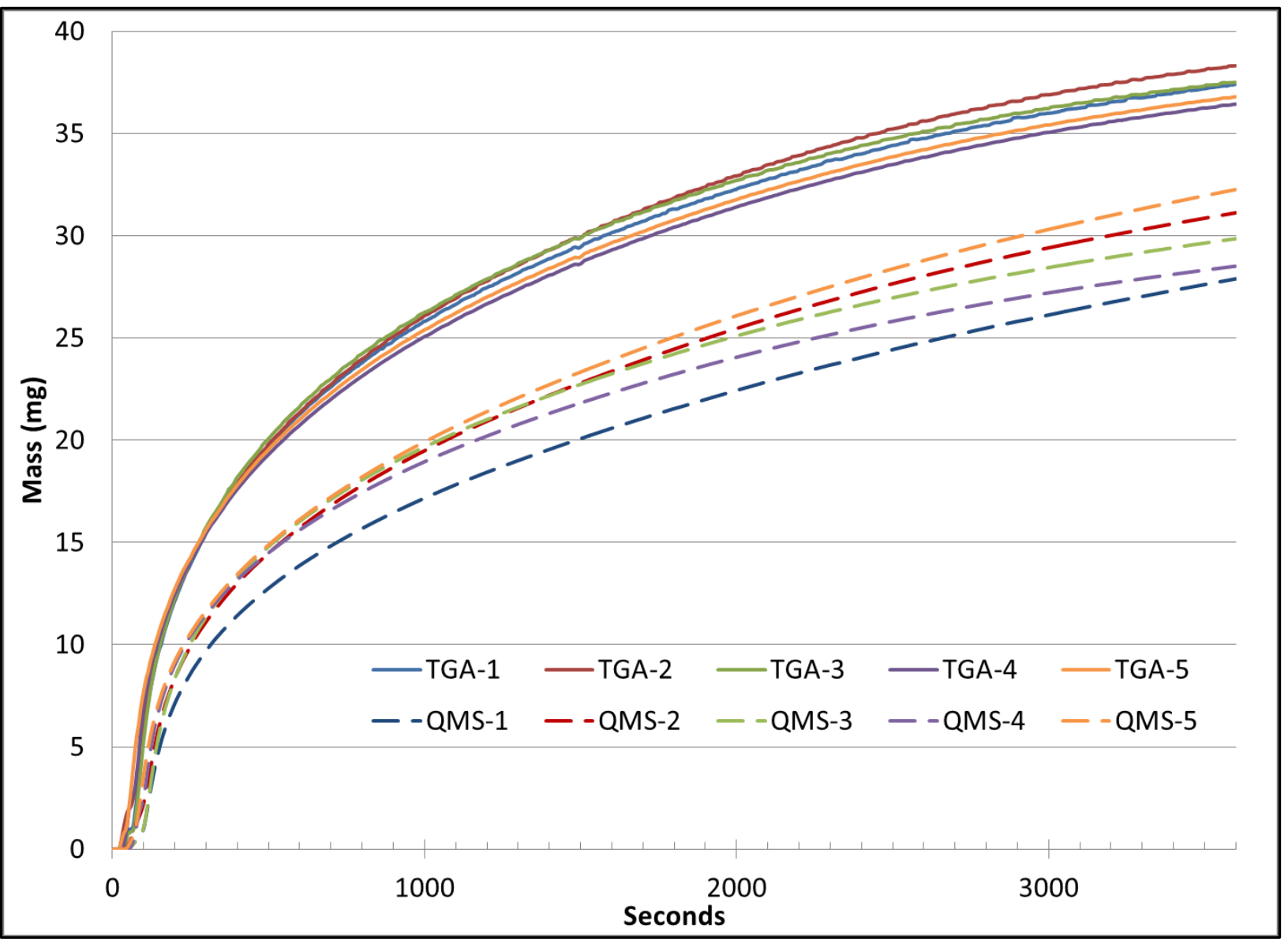

Figure 6: Plot of mass gain as measured through TGA and mass gain as calculated using hydrogen generation data. Zirconium foils were reacted in $1000^{\circ} \mathrm{C}$ steam for one hour.

\subsection{T91 Steel Benchmarking:}

In addition to the zirconium foils, five steam oxidation experiments were performed on T91 steel plates. This was to determine if the technique is material and/or geometry specific. If not, it may be possible to apply the correction factor obtained during the $\mathrm{Zr}$ foil tests to any other system. This would allow one to measure a known standard, determine the correction factor, and then apply it to an unknown system. Similar to the $\mathrm{Zr}$ foil tests, the oxidation rate as measured using TGA was substantially different than that calculated through hydrogen measurement as can be seen in Figure 7. A correction factor may also be applied to the hydrogen measurement data, resulting in reasonable agreement with the TGA data. This correction factor was found to be 1.14, and results in a relative agreement between the two measurement techniques of within $8.4 \%$. This may be seen in Table 2. For the sake of comparison, the relative error among the five T91 steel samples was found to be $11.7 \%$ for TGA and $11.9 \%$ for the hydrogen measurement technique. This high error is almost certainly due to the formation of a spalling oxide which was seen in the T91 steel samples but not in the zirconium foil samples. Interestingly, the error in comparing the two techniques is appreciably lower than that seen among the five samples. The conclusion to be drawn here is that the coupled technique is at least partially able to capture the spallation kinetics.

It was found that the correction factor needed for these T91 samples was equivalent within the margin of error to that seen for the $\mathrm{Zr}$ foil tests. A reduction in the error during hydrogen 
measurement is needed to determine if these two correction factors are indeed equivalent. As mentioned previously, if these factors are different it would be evidence that the hydrogen source term, or the shape and magnitude of the hydrogen pulse produced during steam oxidation, would determine the value of the correction factor. Indeed, it may be seen in Figure 8 that the hydrogen production rate for T91 steel is fundamentally different in both shape and magnitude when compared to that seen for the $\mathrm{Zr}$ foil system.

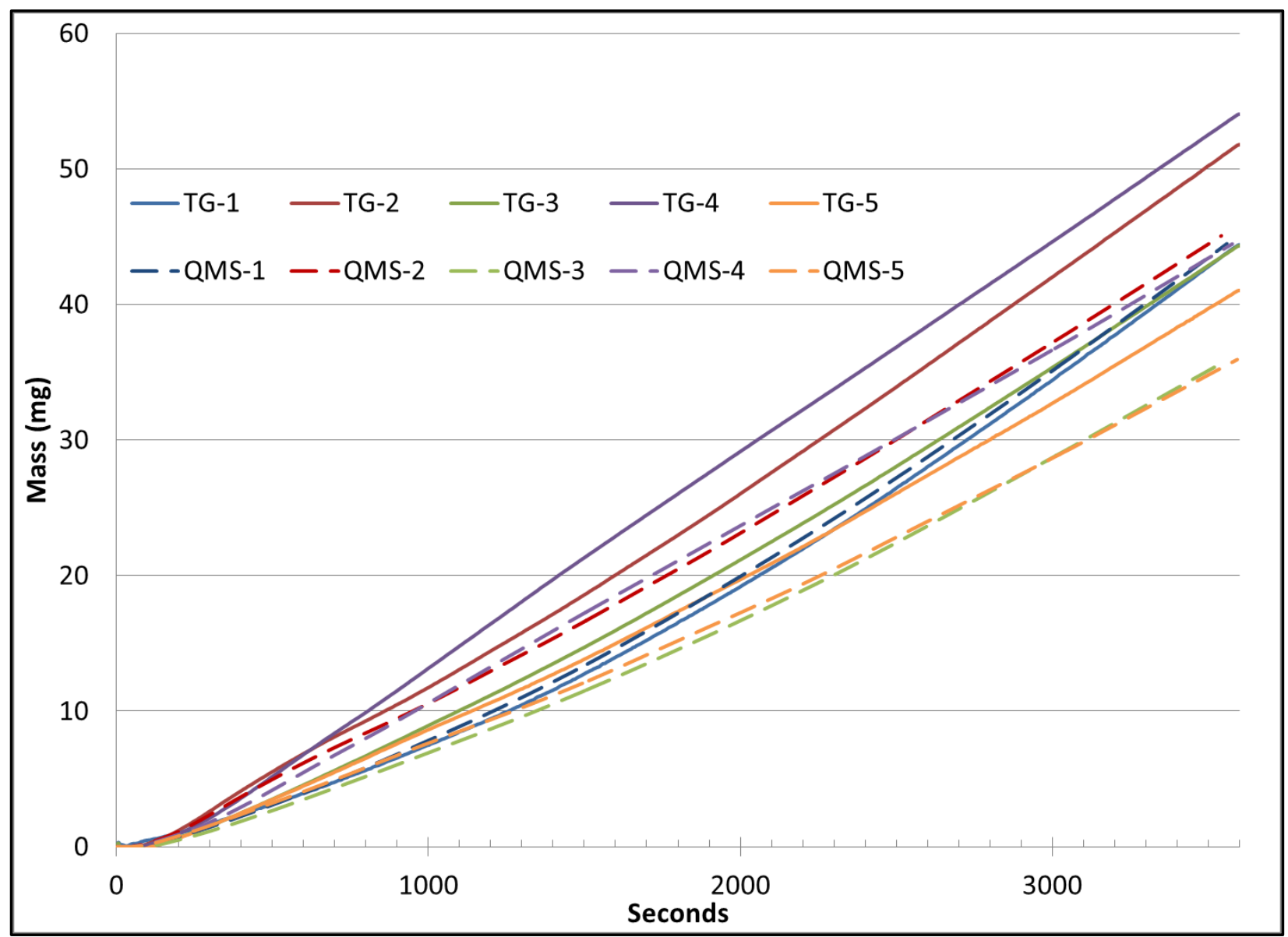

Figure 7: Plot of mass gain as measured through TGA and mass gain as calculated using hydrogen generation data. T91 steel plates were reacted in $1000{ }^{\circ} \mathrm{C}$ steam for one hour. 


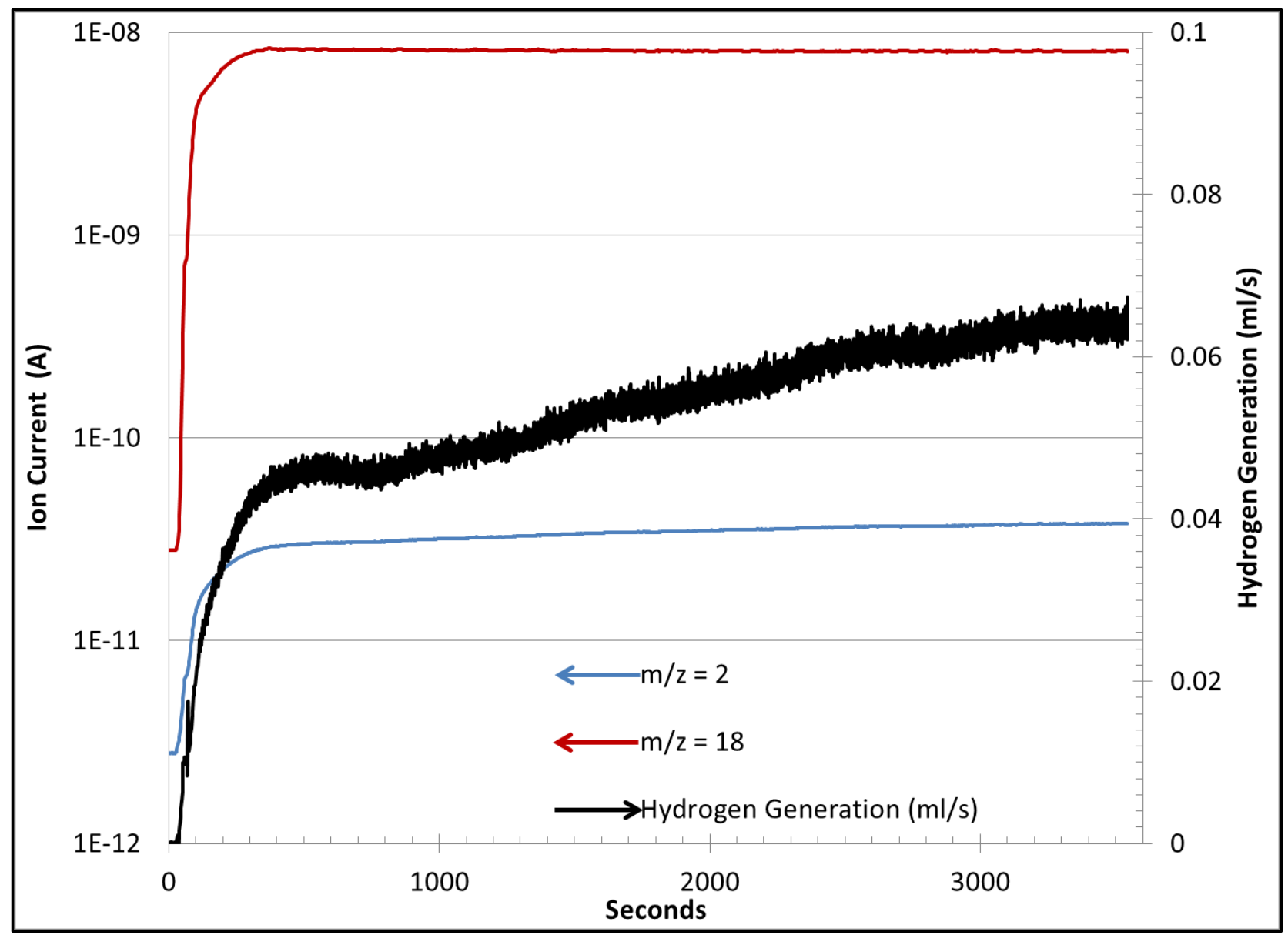

Figure 8: Ion currents for $\mathrm{m} / \mathrm{z}$ of 2 and 18 seen alongside the hydrogen generation rate as determined through QMS measurement. Displayed data is the average of the five T91 steel plate samples reacted in $1000{ }^{\circ} \mathrm{C}$ steam for one hour.

Table 2: Tabulated comparison between mass gains measured using TGA and those calculated using hydrogen generation data. T91 plates were reacted in $1000^{\circ} \mathrm{C}$ steam for one hour.

\begin{tabular}{ccccc}
\hline Sample & $\begin{array}{c}\text { Starting Mass } \\
(\mathrm{mg})\end{array}$ & $\begin{array}{c}\text { TGA Mass Gain } \\
(\mathrm{mg})\end{array}$ & $\begin{array}{c}\text { Hydrogen Calculation } \\
(\mathrm{mg})\end{array}$ & $\begin{array}{c}\text { Correction } \\
\text { Factor }\end{array}$ \\
\hline T91-1 & 1088.21 & 44.40 & 45.0 & 0.99 \\
\hline T91-2 & 1085.99 & 51.79 & 45.6 & 1.13 \\
\hline T91-3 & 1057.14 & 44.33 & 36.1 & 1.23 \\
\hline T91-4 & 1092.61 & 54.04 & 44.5 & 1.22 \\
\hline T91-5 & 1058.37 & 41.04 & 35.9 & 1.14 \\
\hline Mean & & 47.12 & 41.4 & $1.14 \pm 0.10$
\end{tabular}




\section{Conclusions:}

A framework has been laid out which allows any quantitative gas measuring technique to be coupled to TGA measurements, thus providing the researcher access to information which would otherwise be difficult or impossible to obtain for steam oxidation reactions. It is important to note that the provided framework is not necessarily limited to steam oxidation reactions. Indeed, it may be applied to any system containing multiple species in which one of those species produces an interfering fragmentation pattern. An attempt was made to use QMS as the quantification technique during steam oxidation reactions with limited success. Some of the possible sources of error and areas for possible improvement are presented below:

- Flow Rates: The flow rates may be adjusted to increase repeatability of the hydrogen measurement. This will require optimization of the timing between QMS and TGA while being mindful that an increased flow rate will result in a decreased hydrogen signal.

- System Purity: The system used for this investigation had previously been used for molybdenum oxidation experiments. There were indications of a reducible oxide, namely $\mathrm{MoO}_{3}$, being present in the TGA equipment. This may have affected the repeatability of the experiments presented in this paper.

- Scan Time: There remains room for optimization of the scan time within the QMS software. A scan time of one second was used here, but this was not optimized. A longer scan time will increase the precision of the specific $\mathrm{m} / \mathrm{z}$ being scanned, but will decouple the $\mathrm{m} / \mathrm{z}$ signals when applied to the $18 / 2$ ratio. A balance must be reached.

- Peak Resolution: The peak resolution may be adjusted to increase the counts per scan, and thus, increase the precision of the measured signal. A peak resolution of 50 was used here with the idea that a lower peak resolution would decrease the error incurred in measuring trace levels of hydrogen. The counter argument to using a low peak resolution is that any shift in peak location will have an exaggerated effect on the measured signal.

- Geometry Issues: It may be possible to design a system with a shorter and more reliable path for the steam to travel prior to reaching both the sample as well as the gas quantification system.

- Measurement Technique: It may be beneficial to remove the turbo pump efficiency as a variable, possibly through direct sampling of the reaction chamber contents. This may be accomplished by locating the mass spec ionization source within, or at least at the same pressure as, the reaction chamber. Or by using a different measuring technique altogether such as infrared spectroscopy. 


\section{Acknowledgements}

This work was supported by the U.S. Department of Energy, Office of Nuclear Energy Fuel Cycle Research and Development program. 


\section{References}

1. J.D. Higgs, B.J. Lewis, W.T. Thompson, and Z. He (2007). A Conceptual Model for the Fuel Oxidation of Defective Fuel. Journal of Nuclear Materials, 99-128.

2. X. Hu, K.A. Terrani, and B.D. Wirth (2014). Hydrogen Desorption Kinetics from Zirconium Hydride and Zirconium Metal in Vacuum. Journal of Nuclear Materials, 87-95.

3. S. Leistikow, and G. Schanz (1987). Oxidation Kinetics and Related Phenomena of Zircaloy-4 Fuel Cladding Exposed to High Temperature Steam and Hydrogen-Steam Mixtures Under PWR Accident Conditions. Nuclear Engineering and Design, 65-84.

4. F. Nagase, T. Otomo, and H. Uetsuka (2003). Oxidation Kinetics of Low-Sn Zircaloy-4 at the Temperature Range from 773 to 1,573 K. Journal of Nuclear Science and Technology, 213-219.

5. R.E. Pawel (1974). Oxygen Diffusion in Beta Zircaloy during Steam Oxidation. Journal of Nuclear Materials, 247-258.

6. B.A. Pint, K.A. Terrani, M.P. Brady, T. Cheng, and J.R. Keiser (2013). High Temperature Oxidation of Fuel Cladding Candidate Materials in Steam-Hydrogen Environments. Journal of Nuclear Materials, 420-427.

7. W.A. Bostrom (1954). The High Temperature Oxidation of Zircaloy in Water. Pittsburg, Pa: WAPD-104, Westinghouse Electric Corporation.

8. A. Couet, A.T. Motta, and R.J. Comstock (2014). Hydrogen Pickup Measurements in Zirconium Alloys: Relation to Oxidation Kinetics. Journal of Nuclear Materials, 1-13.

9. A.W. Lemmon (1957). Kinetics of the High Temperature Oxidation of Zirconium. Columbus, Oh: BMI-1154, Battelle Memorial Institute.

10. M. Moalem, and D.R. Olander (1991). Oxidation of Zircaloy by Steam. Journal of Nuclear Materials, 170-194.

11. V.F. Urbanic, and T.R. Heidrick (1978). High-Temperature Oxidation of Zircaloy-2 and Zircaloy-4 in Steam. Journal of Nuclear Materials, 251-261.

12. R.E. Westerman (1964). High-Temperature Oxidation of Zirconium and Zircaloy-2 in Oxygen and Water Vapor. Journal of the Electrochemical Society, 140-147.

13. A.T. Nelson, E.S. Sooby, Y.J. Kim, B. Cheng, and S.A. Maloy (2013). High Temperature Oxidation of Molybdenum in Water Vapor Environments. Journal of Nuclear Materials, 441-447.

14. Z M. Steinbruck (2009). Prototypical experiments relating to air oxidation of Zircaloy-4 at high temperatures. Journal of Nuclear Materials, 531-544.

15. J. Belle and M.W. Mallett (1954). Kinetics of the High Temperature Oxidation of Zirconium. Journal of the Electrochemical Society, 339-342.

16. G.R. Wallwork, W.W. Smeltzer, and C.J. Rosa (1964). The Parabolic Oxidation Kinetics of AlphaZirconium at 850 C. ACTA Metallurgica, 409-415.

17. A.J. Parkison and S.M. McDeavitt (2010). Hydride Formation Process for the Powder Metallurgical Recycle of Zircaloy from Used Nuclear Fuel. Metallurgical and Materials Transactions A, 192-201.

18. G.R. Belton and A.S. Jordan (1965). The Volatilization of Molybdenum in the Presence of Water Vapor. The Journal of Physical Chemistry, 2065-2071.

19. D. Wajszel (1963). A Method for Calculating Paralinear Constants for the Formation of Volatile Scale. Journal of the Electrochemical Society, 504-507.

20. M. Kilpatrick and S.K. Lott (1964). Reaction of Flowing Steam with Refractory Metals. I. Molybdenum (1100-1700 ${ }^{\circ}$. Argonne National Laboratory, 1638-1640. 